Curr Opin Neurol. 2014 June ; 27(3): 315-324. doi:10.1097/WCO.0000000000000091.

\title{
Migraine and estrogen
}

\author{
Nu Cindy Chai ${ }^{\mathrm{a}}$, B. Lee Peterlin ${ }^{\mathrm{a}}$, and Anne H. Calhoun ${ }^{\mathrm{b}}$
}

aDepartment of Neurology, Johns Hopkins University School of Medicine, Baltimore, Maryland ${ }^{b}$ Carolina Headache Institute, University of North Carolina, Chapel Hill, North Carolina, USA

\begin{abstract}
Purpose of review-The aim is to systematically and critically review the relationship between migraine and estrogen, the predominant female sex hormone, with a focus on studies published in the last 18 months.

Recent findings-Recent functional MRI (fMRI) studies of the brain support the existence of anatomical and functional differences between men and women, as well as between participants with migraine and healthy controls. In addition to the naturally occurring changes in endogenous sex hormones over the lifespan (e.g. puberty and menopause), exogenous sex hormones (e.g. hormonal contraception or hormone therapy) also may modulate migraine. Recent data support the historical view of an elevated risk of migraine with significant drops in estrogen levels. In addition, several lines of research support that reducing the magnitude of decline in estrogen concentrations prevents menstrually related migraine (MRM) and migraine aura frequency.

Summary-Current literature has consistently demonstrated that headache, in particular migraine, is more prevalent in women as compared with men, specifically during reproductive years. Recent studies have found differences in headache characteristics, central nervous system anatomy, as well as functional activation by fMRI between the sexes in migraine patients. Although the cause underlying these differences is likely multifactorial, considerable evidence supports an important role for sex hormones. Recent studies continue to support that MRM is precipitated by drops in estrogen concentrations, and minimizing this decline may prevent these headaches. Limited data also suggest that specific regimens of combined hormone contraceptive use in MRM and migraine with aura may decrease both headache frequency and aura.
\end{abstract}

\section{Keywords}

estradiol; estrogen; headache; migraine; sex hormone 


\section{INTRODUCTION}

Substantial research has emerged in the recent years highlighting sex differences in the epidemiology and characteristics of various headache disorders, specifically in migraine. The cause underlying these sex differences is likely multifactorial, involving both biological and psychosocial factors. Of the many biological factors, sex hormones are likely a major contributor. In addition to differences between men and women, migraine prevalence rates and characteristics vary in women by hormonal status (i.e. across the menstrual cycle, with or without taking hormonal contraceptives), further underscoring a strong role for sex hormones in headache.

Although the association between migraine and sex hormones has been repeatedly demonstrated, the exact pathophysiology of this association has not yet been fully elucidated. Further, for many clinicians, the effect of various hormonal contraceptive agents on migraine, specifically migraine with aura (MwA) continues to be a source of confusion. In this review, we first briefly discuss the most recent epidemiologic data evaluating sex differences in migraine. We then systematically and critically review the available clinical and experimental studies published in the last 18 months examining the role of estrogen, the predominant female sex hormone, in migraine. We close with a brief discussion on future directions necessary to further elucidate the role of estrogen and sex hormones in migraine.

\section{SEARCH METHOD}

A systematic search of the PubMed database was conducted using keywords 'estrogen', 'estradiol', 'estrone' or 'sex hormone' and 'headache', 'pain' or 'migraine'. In addition, reference lists of relevant articles were reviewed for inclusion. General population studies (cross-sectional and longitudinal), clinic-based case series, human clinical trials as well as animal studies evaluating the association between estrogen and migraine were included. Studies evaluating the effect of sex hormones on pain in general, headache in general or other headache subtypes were excluded. Only studies published between April 2012 and October 2013 were included for discussion in this article.

\section{SEX DIFFERENCES IN HEADACHE: EPIDEMIOLOGIC STUDIES}

An expansive, world-wide, body of literature has consistently demonstrated that headache, and in particular migraine, is more prevalent in women as compared with men [1-3]. Most recently, Buse et al. [4] analyzed data from over 160000 participants, 12 years of age or older, in the American Migraine Prevalence and Prevention study and again substantiated the previously reported higher prevalence of migraine in women as compared with men at all ages. The female to male ratio peaked at 3.25 among those between 18 and 29 years of age [probability ratio $3.25 ; 95 \%$ confidence interval (CI): 3.00, 3.53]. Similarly, probable migraine was also found to be more prevalent in women (peaking at ages 18-29, probability ratio $1.53,95 \% \mathrm{CI}=1.35-1.73$ ) as compared with men. As previously described [1], this female predominance was consistent across racial groups [4]. Further, this study reported that although men and women reported similar headache severity and frequency, women reported more migraine-related symptoms (i.e. photophobia, nausea, etc) and more migraine-related disability [4]. 


\section{SEX DIFFERENCES IN HEADACHE: IMAGING STUDIES}

In addition to epidemiologic evidence of sex differences in migraine, brain MRI studies support both structural and functional sex differences in those with migraine [5"].

Specifically, Maleki et al. [5"] used high-field MRI to compare age-matched male and female migraineurs to healthy controls. Female migraineurs were found to have thicker posterior insula and precuneus cortices as compared with both male migraineurs and healthy controls of both sexes. However, no difference in cortical thickness was found between male migraineurs and male healthy controls. Furthermore, using functional MRI (fMRI), noxious thermal stimulation produced stronger responses in areas such as the amygdala and parahippocampus, in female as compared with male participants with migraine.

Maleki et al.'s study is of particular interest as the anatomical difference found in the female migraineur is contributable neither to the female sex alone nor the migraine disease state alone (as it was compared with both female healthy controls and male participants with migraine). Therefore, one can theorize that a combination of being female and having migraine may lead to changes in brain anatomy and sensory responsiveness and result in differences in migraine characteristics, (as seen in epidemiological studies). However, as Maleki's study cannot determine the directionality of the association between the anatomical difference among the sexes and the migraine disease state, it is also possible that those with a specific anatomical variant (i.e. thicker posterior insular and precuneus cortices) together with being of the female sex (and encompassing female-specific levels and ratios of sex hormones) can account for an increased risk of developing migraine.

\section{SEX HORMONE'S CONTRIBUTION IN MIGRAINE}

The above data suggest that although both men and women may suffer from migraine, 'male migraine' and 'female migraine' differ in characteristics because of their difference in sex, which is most obviously marked by sex hormone differences. In addition, the higher migraine prevalence among women of reproductive age, and the lower migraine prevalence among those prior to puberty and postmenopausal may also be, at least in part, explained by their different sex hormone status.

Overall, research in the area of sex hormone and headache is active and diverse, including both animal and human studies. Estrogen, a predominant female sex hormone, is of particular interest. In the next section, we briefly discuss the role of estrogen in the central nervous system (CNS), and critically review the most recent studies evaluating its implication in migraine.

\section{ESTROGEN AND THE CENTRAL NERVOUS SYSTEM}

In nonpregnant women, estrogens are produced primarily by the ovaries, and in smaller quantities by adipocytes, the liver, adrenal glands and breasts. In men, estrogen is synthesized from its precursors, androstenedione and testosterone, by aromatization. Although estrogen quantities are much lower in men than in young women, they are relatively stable in men and undergo a much more gradual decline with age as compared with women - to the extent that men over the age of 60 have approximately three times 
more circulating estradiol than postmenopausal women who are not taking exogenous estrogen [6]. In addition to reproduction, accumulating data support that estrogen participates in multiple physiological and pathological processes in both sexes, including homeostasis, memory and pain processing [7-9].

In nonpregnant women, the three major estrogens are estradiol (E2), estrone and estriol, with estradiol being the most potent form [10]. Estrogen's effect on the brain can occur via at least three distinct pathways: direct diffusion of peripheral estrogen into the CNS, conversion of testosterone and androstenedione in the brain by aromatases in the presynaptic terminals in the brain [11] or de-novo synthesis of estrogen in the brain from cholesterol [12]. In the brain, estrogen can exert its effect by binding to estrogen receptors, usually located in the nucleus or cytoplasm, with subsequent gene transcription and protein synthesis; or alternatively estrogen can act by nongenomic mechanisms [13]. Recent data suggest that the transcriptional activity of human brain estrogen receptors is cell-type specific [14].

\section{ANIMAL STUDIES}

Many limitations exist in animal studies evaluating migraine. First, the majority of studies use predominantly male animals, which may not be representative of a predominantly female disorder $[15,16]$. Secondly, despite a variety of different migraine animal models, none are able to fully model the complexities of the various pain and pain-related symptoms of migraine. Finally, most animal models of migraine have been formed based on our current understanding of migraine pathophysiology, and rely less on the nociceptive behavior of the animals to corroborate that headache was the result of the interventions [17]. With these limitations in mind, two studies using two different rodent models of migraine were recently published and are reviewed below (see Table 1) $[18,19]$.

Using the nitroglycerin (NTG)-induced neuronal activation (as detected by fos activation) rodent migraine model, Greco et al. [18] attempted to evaluate the influence of estrogen on migraine in rats. Intact females were tested during the proestrus phase of the estrus cycle (generally corresponding to a period of estrogen peak, see Fig. 1a) [20], and were demonstrated to have an increased NTG-induced activation in the paraventricular nucleus and supraoptic nucleus of the hypothalamus, as well as the nucleus trigeminalis caudalis of the brainstem as compared with intact male rats. Further, although ovariectomy significantly reduced neuronal activation in all above-mentioned areas in the female rat, chronic intraperitoneal administration of estrogen restored the activation levels to those of an intact female [18]. In contrast, in male gonadectomized rats, neuronal suppression was demonstrated only in the nucleus trigeminalis caudalis as compared with intact males.

In a second study, using the migraine rodent model of meningeal inflammation as measured by dural mast cell activation, Boes and Levy [19] demonstrated that dural mast cell activation in intact female rats was consistently higher than in intact males, with the exception of the proestrus phase (i.e. peak estrogen levels). In addition, overall mast cell density was higher in estrus (a period of somewhat lower estrogen concentrations, see Fig. 1a) $(P<0.01)$ and even higher in diestrus 1 (when estrogen concentrations are low) $(P$ 
$<0.01$ ) as compared with the proestrus (peak estrogen) or the diestrus 2 (rising estrogen) phases. Further, although ovariectomized females had decreased mast cell density, those ovariectomized females that received a subsequent subcutaneous injection of E2 showed an increase in dural mast cell density compared with those treated with placebo. Interestingly, administration of progesterone together with E2 blunted the increase in dural mast cell density seen after E2 treatment alone. Boes and Levy [19] also reported that the effect of E2 on mast cell density was time-specific - being apparent at 24 and $48 \mathrm{~h}$ after E2 injection and disappearing after $72 \mathrm{~h}$.

The above studies appear to report contradictory findings. Greco et al. [18] demonstrated elevated pain sensitivity in females (increased NTG-induced fos activation) corresponding to the proestrus phase, whereas Boes and Levy [19] showed a relatively lower state of painsensitivity (decreased mast cell activation) in proestrus. Further, even though Boes and Levy showed a lower pain response during a phase of high estrogen (proestrus) in normal cycling rats, they showed an increased pain response when subcutaneous estrogen was given to animals of a low estrogen state (ovariectomized rats). These conflicting data may be largely because of the difficulty in determining the specific hormonal levels in rats (Fig. 1a). Notably, neither study directly evaluated the status of sex hormones in the animals (i.e. obtaining serum estrogen levels), but instead determined the estrus cycle based on vaginal smears, a method extremely variable depending on the specific timing of the studies (Fig. 1). These studies also demonstrate the difficulty in extrapolating the results of animal studies to humans, as rats have brief 4-day estrus cycles in which a difference of a few hours can produce either peak or trough estrogen concentrations (Fig. 1).

In addition to estrogen itself, different estrogen receptors have been studied recently in animal models of general pain, although not specifically in headache or migraine [21-23]. As the differential effect of estrogen may very well be receptor-specific, further studies on estrogen receptors in the headache or migraine rodent models would be of interest.

\section{HUMAN STUDIES}

Most human studies evaluating the effect of sex hormones on migraine have employed either the documentation of life events marked by endogenous hormonal changes (e.g. menstruation and menopause) or exogenous hormone use [e.g. contraceptive medication and hormone replacement therapy (HRT)] to simulate a controlled hormonal state (Table 2) $\left[24,25,26^{\mathbf{*}}\right]$. One of the most significant limitations to these studies is the inability to truly control each participant's hormonal state. Specifically, the effect of hormonal contraceptives on the total hormone level is significantly different depending on the pharmacology and the 'architecture' of the contraceptive administered, as well as whether ovulation was successfully inhibited. Most combined hormonal contraceptives (CHCs) allow for a drop in ethinyl estradiol concentration during a placebo week that ranges from 20 to $50 \mu \mathrm{g}$, levels sufficient to trigger an estrogen withdrawal migraine. Only two regimens limit this drop to $10-15 \mu \mathrm{g}$, and would, therefore, not be expected to trigger menstrually related migraine (MRM) [27]. Further, although CHCs almost always result in higher estrogen concentrations than the native menstrual cycle, if ovulation is inhibited with one of the very few ultra-low dose $\mathrm{CHCs}$ the resulting estrogen concentration can actually be lower than that of the native 
menstrual cycle. Thus, it is important for clinicians examining the available data to be mindful of the type and dose of hormone therapy in order to draw appropriate conclusions. Additionally, the relationship between different sex hormones (i.e. estrogens, progestogens and testosterone) is complex and should be taken into consideration as a whole, in order to properly evaluate the role of sex hormones in headache. And finally, most studies evaluate only a small number of individuals, limiting our ability to draw firm conclusions.

\section{Studies with a focus on changes in endogenous sex hormones}

One recent cross-sectional survey evaluated the effect of endogenous hormonal changes in women with migraine vs. tension-type headache (TTH). In this study, Karli et al. [28] conducted a general population observational study evaluating 1333 women aged 18-65. Participants responded to questions regarding menstruation, pregnancy, oral contraception intake, as well as menopause and their impact on headache. Those with migraine were found to be almost three-fold more likely to experience headache during menstruation than those with TTH (odds ratio 2.79; 95\% CI: 1.33-5.85, $P=0.006$ ) [28]. Additionally, although previous data suggested a protective effect of pregnancy, in particular during the third trimester, for those women with migraine [29], pregnancy did not appear to affect migraine or TTH characteristics in any specific trend in this study. In addition, although the type and dose of oral contraceptives used was not reported (a methodological limitation which severely limits the conclusions that may be drawn from this aspect of the study) oral contraceptive use was reported to be more likely to worsen migraine compared with TTH (39 vs. 3.6\%, $P<0.001$ ). Finally, more participants with migraine than those with TTH had improvement in their headache during menopause (15.8 vs. $2.4 \%, P=0.046)$ [28].

In summary, although this study provides few new clues to the hormonal differences in different headache conditions, it substantiates and highlights that participants with migraine have a higher sensitivity to varying hormonal levels as compared with those with TTH. Further, menopause, a life event marked by stable ovarian estrogen production (although very low), generally corresponds to improvement in migraine.

\section{Studies with a focus on the effect of exogenous sex hormones}

Exogenous sex hormones (e.g. combined contraceptives) can also affect headache. Specifically, estrogen administration in male-to-female transsexuals has been linked to the development and worsening of headache [30] (Dr Thomas N. Ward, personal communication). Further, abrupt drops in estrogen have long been suspected as a migraine trigger [31]. On the basis of the observation of the existence of headache with estrogen withdrawal, two recent studies evaluated the effect of estradiol valerate/dienogest (E2V/ DNG) on headache [24,25]. (Note: E2V/DNG is a tetraphasic - 2/5/17/2/2 regimen combination oral contraceptive administered in an estrogen step-down and progestin step-up regimen. Each decline in estrogen is limited to $1 \mathrm{mg}$ E2V, a decline equal to that originally proposed by Calhoun [27] to prevent MRM.)

Both studies demonstrated a reduction in headache frequency and headache-medication usage in participants using E2V/DNG [24,25] (see Table 2). Specifically, in a multicenter, randomized, double-blinded study, Macias et al. [24] evaluated 223 women aged 18-50 who 
received E2V/DNG for six cycles, while a control group of 218 women received a traditional 21/7 regimen of ethinyl estradiol/levonorgestrel (ethinyl estradiol $20 \mu \mathrm{g} / \mathrm{LNG} 100$ $\mu \mathrm{g})$. E2 V/DNG reduced the frequency and intensity of headache from baseline when evaluated at cycle six (change from baseline: $47.7 \pm 29.4 \mathrm{~mm}$ ) [24]. E2 V/DNG was also associated with a greater mean reduction in the use of rescue medication on days 22-28, compared with ethinyl estradiol/LNG by cycle six (number of ibuprofen $200 \mathrm{mg}$ tablets: 3.5 \pm 6.3 vs. $1.8 \pm 6.0$ tablets; $95 \%$ CI: $3.04-0.48 ; P<0.05$ ) [24]. Interestingly, investigators reported that to a lesser degree, those randomized to the ethinyl estradiol $20 \mu \mathrm{g} / \mathrm{LNG} 100 \mu \mathrm{g}$ regimen also improved in their headache intensity at the end of the study from baseline (34.5 $\pm 25.7 \mathrm{~mm}$ ). This would be expected if the participants at baseline were taking a combined oral contraceptive (COC) with ethinyl estradiol dose greater than $20 \mu \mathrm{g}$, as the decline in estrogen concentration on those pills would be $25-50 \mu \mathrm{g}$, greater than the $20 \mu \mathrm{g}$ decline with the study drug. However, as the investigators did not report baseline COC estrogen doses, this cannot be accurately measured.

In a second prospective clinic-based study, 32 women older than 35 years of age with the diagnosis of MRM were included [25]. The number of migraine attacks, duration as well as severity of the head pain were all significantly reduced at the third and sixth cycles of E2 V/DNG compared with baseline [25]. In addition, and similar to the findings reported by Macias et al. [24], a significantly lower number of acute abortive analgesics were reported at cycle six $(4.7 \pm 1.1-2.9 \pm 0.6, P<0.001)[25]$.

Overall, these studies demonstrate that sex hormones, both endogenous and exogenous, can cause alterations in headache patterns in participants with migraine [24,25]. The avoidance of large magnitude drops in estrogen may improve MRM or other estrogen withdrawal migraines.

In addition to the pain of migraine, aura has also been demonstrated to vary with estrogen levels, being more prevalent with high estrogen concentrations and absent in the low estrogen environment of MRM [26"']. And of more concern, in some studies, migraine's increased stroke risk parallels the frequency of aura [32-34]. Calhoun et al. [26"'] conducted a retrospective, clinic-based case series evaluating 23 women with MwA and MRM, and observed a reduction in aura frequency after extended cycle dosing of a transvaginal ring contraceptive. This dosing delivers $15 \mu \mathrm{g}$ ethinyl estradiol $/ 0.120 \mathrm{mg}$ etonogestrel per $24 \mathrm{~h}$. Extended cycle use was prescribed to assure inhibition of ovulation with this ultra low-dose $\mathrm{CHC}$, thereby creating an estrogen environment lower than the native menstrual cycle. Median aura frequency was reduced from 3.23 to 0.23 after treatment $(P<0.0005)$. Investigators found significantly improved $\mathrm{MwA}(\mathrm{Z}=-4.58, P<0.0005)$ as well as MRM (headache eliminated in $91.3 \%$ of the evaluable individuals) after treatment [26"'].

\section{Genetic studies}

Although the above studies focused on the pain and associated symptoms of migraine (i.e. aura) in those with different hormonal states, one recent study evaluated the genetic association between estrogen receptor and migraine. Rodriguez-Acevedo et al. [35] recently evaluated the genetics of the Norfolk Island population, an isolated population with high levels of consanguineous unions and a relatively homogenous lifestyle, as well as a high 
migraine prevalence. Serum studies and questionnaires were collected from 600 individuals, and 76 participants with migraine were identified. As previous studies have mapped genes associated with migraine risk in this population [36], Rodriguez-Acevedo et al. [35] identified an association with migraine in at least 10 markers of the estrogen receptor 1 gene. Together with recent data revealing substantial heritability in the circulating levels of sex hormones in adult men [37], these data add complexity to the contribution of estrogen in the heritability of migraine in certain populations.

\section{THERAPEUTIC CONSIDERATIONS AND FUTURE DIRECTIONS}

Although accumulating evidence supports a role for sex hormones in headache, and particularly migraine, much about the specific mechanisms of how sex hormones affect migraine remains unknown. As estrogen can extend its impact in a variety of systems, its role in headache is likely multifactorial, including direct cellular and genetic modifications in the CNS, as well as potentially as a result of its effect on mood and pain perception $[13,38,39]$. Furthermore, the relationship between various sex hormones (i.e. estrogens and testosterone) is complex, and future studies focusing on the association between these sex hormones and headache must take into account these interactions.

In addition to pain, migraine aura is affected by sex hormones. Although human studies have demonstrated a reduction of aura with extreme low-dose CHC [31], experts stress the importance of inhibition of ovulation as a critical precursor to reduction of aura (so that the ultra-low doses of these new regimens do not simply augment the endogenous fluctuating hormone levels). As many women are denied therapy with CHCs, in part because of published guidelines from the American Congress of Obstetricians and Gynecologists (ACOG) that recommend against their use in migraine with aura, as well as the lack of formal statement or guidelines from the International and American Headache Societies, Calhoun et al.'s recent study demonstrating decreased aura frequency with one of the new ultra-low-dose regimens may: point to more individualized treatment plans; stimulate larger, confirmatory trials to establish the safety of $\mathrm{CHC}$ use in MwA; and open discussions between the American and International Headache Societies and ACOG on revisions of ACOG's published guidelines.

\section{CONCLUSION}

Current literature has consistently demonstrated that headache, in particular migraine, is more prevalent in women as compared with men, specifically during reproductive years. Recent studies have found differences in headache characteristics, CNS anatomy as well as functional activation by fMRI between the sexes in migraine patients. Although the cause underlying these differences is likely multi-factorial, considerable evidence supports an important role for sex hormones. Recent studies continue to support that MRM is precipitated by drops in estrogen concentrations, and minimizing this decline may prevent these headaches. Limited data also suggest that specific regimens of CHC use in MRM and MwA may decrease both headache frequency and aura. 
The role of female sex hormone in migraine is continuing to unfold. Future research to firmly establish the role of CHC and HRT in those with migraine, including those with aura, is still needed. Additionally, studies with particular focus on delineating the role and complex relationships between different sex hormones (i.e. estrogen, progesterone/progestin and testosterone) in headache would be of particular interest. Although translational and basic science studies have advanced our understanding of the mechanisms of sex hormones, many questions remain, and our understanding of this topic continues to evolve.

\section{Acknowledgments}

Note: in this article, the term menstrually related migraine (MRM) also encompasses estrogen withdrawal migraines, such as those resulting from some combined hormonal contraceptives.

B.L.P. receives investigator initiated research support from GSK and Luitpold Pharmaceuticals for studies unrelated to the current article, royalties from Oxford University Press, funding by NIH/NINDS grant \# K23-NS078345, a Landenberger Foundation grant for a study unrelated to the current article, and serves as an associate editor for the journals Headache and BMC Neurology. A. C. receives research support from GSK and ElectroCore for studies unrelated to the current article, serves on Advisory Boards of Allergan, Nautilus and Zogenix. N.C.C. has no disclosures.

\section{REFERENCES AND RECOMMENDED READING}

Papers of particular interest, published within the annual period of review, have been highlighted as:

- of special interest

- of outstanding interest

1. Stewart WF, Lipton RB, Celentano DD, Reed ML. Prevalence of migraine headache in the United States. Relation to age, income, race, and other sociodemographic factors. JAMA. 1992; 267:64-69. [PubMed: 1727198]

2. Lipton RB, Stewart WF, Diamond S, et al. Prevalence and burden of migraine in the United States: data from the American Migraine Study II. Headache. 2001; 41:646-657. [PubMed: 11554952]

3. Stovner L, Hagen K, Jensen R, et al. The global burden of headache: a documentation of headache prevalence and disability worldwide. Cephalalgia. 2007; 27:193-210. [PubMed: 17381554]

4. Buse DC, Loder EW, Gorman JA, et al. Sex differences in the prevalence, symptoms, and associated features of migraine, probable migraine and other severe headache: results of the American migraine prevalence and prevention (AMPP) study. Headache. 2013; 53:1278-1299. [PubMed: 23808666]

5. Maleki N, Linnman C, Brawn J, et al. Her versus his migraine: multiple sex $\boldsymbol{\bullet}$ differences in brain function and structure. Brain. 2012; 135 (Pt 8):2546-2559. A study using high-field MRI to compare the anatomy and CNS functional reactivity in age-matched male and female migraineurs and healthy controls. This study found that female migraineurs had thicker posterior insula and precuneus cortices as compared with both male migraineurs and healthy controls of both sexes. [PubMed: 22843414]

6. Ferrini RL, Barrett-Connor E. Sex hormones and age: a cross-sectional study of testosterone and estradiol and their bioavailable fractions in community-dwelling men. Am J Epidemiol. 1998; 147:750-754. [PubMed: 9554416]

7. Harte-Hargrove LC, Maclusky NJ, Scharfman HE. Brain-derived neurotrophic factor-estrogen interactions in the hippocampal mossy fiber pathway: implications for normal brain function and disease. Neuroscience. 2013; 239:46-66. [PubMed: 23276673]

8. Cui J, Shen Y, Li R. Estrogen synthesis and signaling pathways during aging: from periphery to brain. Trends Mol Med. 2013; 19:197-209. [PubMed: 23348042] 
9. Asl SZ, Khaksari M, Khachki AS, et al. Contribution of estrogen receptors alpha and beta in the brain response to traumatic brain injury. J Neurosurg. 2013; 119:353-361. [PubMed: 23724987]

10. Baker ME. What are the physiological estrogens? Steroids. 2013; 78:337-340. [PubMed: 23313336]

11. Cornil CA, Ball GF, Balthazart J. Functional significance of the rapid regulation of brain estrogen action: where do the estrogens come from? Brain Res. 2006; 1126:2-26. [PubMed: 16978590]

12. Prange-Kiel J, Rune GM. Direct and indirect effects of estrogen on rat hippocampus. Neuroscience. 2006; 138:765-772. [PubMed: 16324798]

13. Amandusson A, Blomqvist A. Estrogenic influences in pain processing. Front Neuroendocrinol. 2013; 34:329-349. [PubMed: 23817054]

14. Ishunina TA, Sluiter AA, Swaab DF, Verwer RW. Transcriptional activity of human brain estrogen receptor-alpha splice variants: evidence for cell type-specific regulation. Brain Res. 2013; 1500:19. [PubMed: 23333800]

15. Bolay H, Berman NE, Akcali D. Sex-related differences in animal models of migraine headache. Headache. 2011; 51:891-904. [PubMed: 21631475]

16. Peterlin BL, Gupta S, Ward TN, Macgregor A. Sex matters: evaluating sex and gender in migraine and headache research. Headache. 2011; 51:839-842. [PubMed: 21631471]

17. Romero-Reyes M, Ye Y. Pearls and pitfalls in experimental in vivo models of headache: conscious behavioral research. Cephalalgia. 2013; 33:566-576. [PubMed: 23671253]

18. Greco R, Tassorelli C, Mangione AS, et al. Effect of sex and estrogens on neuronal activation in an animal model of migraine. Headache. 2013; 53:288-296. [PubMed: 22913654]

19. Boes T, Levy D. Influence of sex, estrous cycle, and estrogen on intracranial dural mast cells. Cephalalgia. 2012; 32:924-931. [PubMed: 22833613]

20. Emanuele MA, Wezeman F, Emanuele NV. Alcohol's effects on female reproductive function. Alcohol Res Health. 2002; 26:274-281. [PubMed: 12875037]

21. Cao DY, Ji Y, Tang B, Traub RJ. Estrogen receptor beta activation is antinociceptive in a model of visceral pain in the rat. J Pain. 2012; 13:685-694. [PubMed: 22698981]

22. Zhang Y, Lu N, Zhao ZQ, Zhang YQ. Involvement of estrogen in rapid pain modulation in the rat spinal cord. Neurochem Res. 2012; 37:2697-2705. [PubMed: 22903468]

23. Zhang Y, Xiao X, Zhang XM, et al. Estrogen facilitates spinal cord synaptic transmission via membrane-bound estrogen receptors: implications for pain hypersensitivity. J Biol Chem. 2012; 287:33268-33281. [PubMed: 22869379]

24. Macias G, Merki-Feld GS, Parke S, et al. Effects of a combined oral contraceptive containing oestradiol valerate/dienogest on hormone withdrawal-associated symptoms: results from the multicentre, randomised, double-blind, active-controlled HARMONY II study. J Obstet Gynaecol. 2013; 33:591-596. [PubMed: 23919857]

25. Nappi RE, Terreno E, Sances G, et al. Effect of a contraceptive pill containing estradiol valerate and dienogest (E2 V/DNG) in women with menstrually-related migraine (MRM). Contraception. 2013; 88:369-375. [PubMed: 23453784]

26. Calhoun A, Ford S, Pruitt A. The impact of extended-cycle vaginal ring $\boldsymbol{m}$ contraception on migraine aura: a retrospective case series. Headache. 2012; 52:1246-1253. A retrospective case series evaluating women with MwA and MRM. This study observed a reduction in aura frequency after extended cycle dosing of a transvaginal ring contraceptive. [PubMed: 22789073]

27. Calhoun AH. A novel specific prophylaxis for menstrual-associated migraine. South Med J. 2004; 97:819-822. [PubMed: 15455962]

28. Karli N, Baykan B, Ertas M, et al. Turkish Headache Prevalence Study Group. Impact of sex hormonal changes on tension-type headache and migraine: a cross-sectional population-based survey in 2,600 women. J Headache Pain. 2012; 13:557-565. [PubMed: 22935969]

29. Aegidius K, Zwart JA, Hagen K, Stovner L. The effect of pregnancy and parity on headache prevalence: the head-HUNT study. Headache. 2009; 49:851-859. [PubMed: 19545250]

30. Aloisi AM, Bachiocco V, Costantino A, et al. Cross-sex hormone administration changes pain in transsexual women and men. Pain. 2007; 132 (Suppl 1):S60-S67. [PubMed: 17379410] 
31. Somerville BW. Estrogen-withdrawal migraine. I. Duration of exposure required and attempted prophylaxis by premenstrual estrogen administration. Neurology. 1975; 25:239-244. [PubMed: 1167630]

32. Etminan M, Takkouche B, Isorna FC, Samii A. Risk of ischaemic stroke in people with migraine: systematic review and meta-analysis of observational studies. BMJ. 2005; 330:63. [PubMed: 15596418]

33. Schurks M, Rist PM, Bigal ME, et al. Migraine and cardiovascular disease: systematic review and meta-analysis. BMJ. 2009; 339:b3914. [PubMed: 19861375]

34. Spector JT, Kahn SR, Jones MR, et al. Migraine headache and ischemic stroke risk: an updated meta-analysis. Am J Med. 2010; 123:612-624. [PubMed: 20493462]

35. Rodriguez-Acevedo AJ, Maher BH, Lea RA, et al. Association of oestrogen-receptor gene (ESR1) polymorphisms with migraine in the large Norfolk island pedigree. Cephalalgia. 2013; 33:11391147. [PubMed: 23674830]

36. Cox HC, Lea RA, Bellis C, et al. A genome-wide analysis of 'bounty' descendants implicates several novel variants in migraine susceptibility. Neurogenetics. 2012; 13:261-266. [PubMed: 22678113]

37. Travison TG, Zhuang WV, Lunetta KL, et al. The heritability of circulating testosterone, oestradiol, oestrone and sex hormone binding globulin concentrations in men: the Framingham Heart Study. Clin Endocrinol (Oxf). 2014; 80:277-282. [PubMed: 23746309]

38. Bartley EJ, Fillingim RB. Sex differences in pain: a brief review of clinical and experimental findings. Br J Anaesth. 2013; 111:52-58. [PubMed: 23794645]

39. Rettberg JR, Yao J, Brinton RD. Estrogen: a master regulator of bioenergetic systems in the brain and body. Front Neuroendocrinol. 2014; 35:8-30. [PubMed: 23994581] 


\section{KEY POINTS}

- Although one of the most common neurological disorders in both women and men, migraine is $2-3$ times more prevalent in women as compared with men.

- Migraine is most prevalent in those of reproductive age.

- Anatomical and brain functional reactivity differences exist between female and male migraineurs.

- Although steady or increasing levels of estrogen may reduce the risk of migraine (i.e. pregnancy and menopause), acute migraine attacks may be triggered by significant drops in estrogen levels.

- Reducing the magnitude of decline in estrogen concentrations in female migraineurs of reproductive age prevents MRM and reduces MwA attack frequency. 


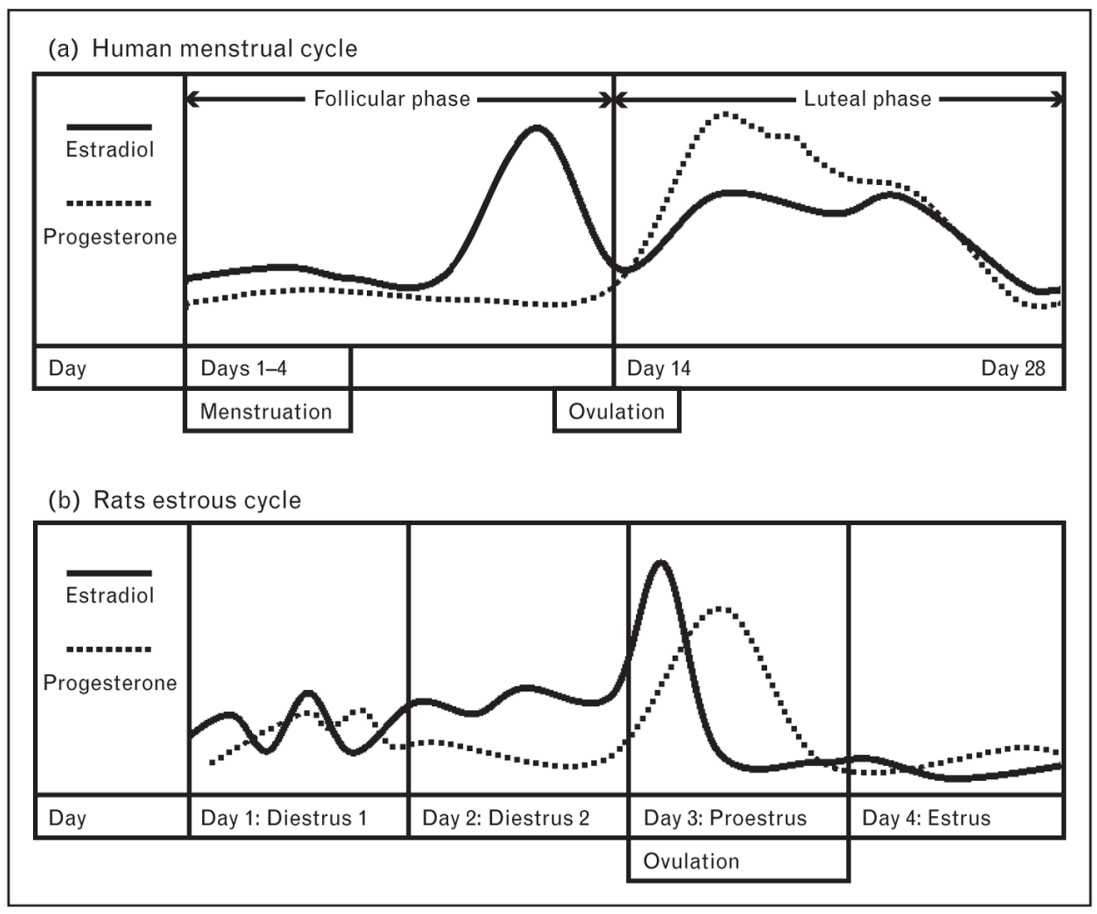

FIGURE 1.

Relative fluctuations of estradiol and progesterone in the human menstrual cycle and rats estrous cycle. Adapted with permission from Figure 3 of [20]. 


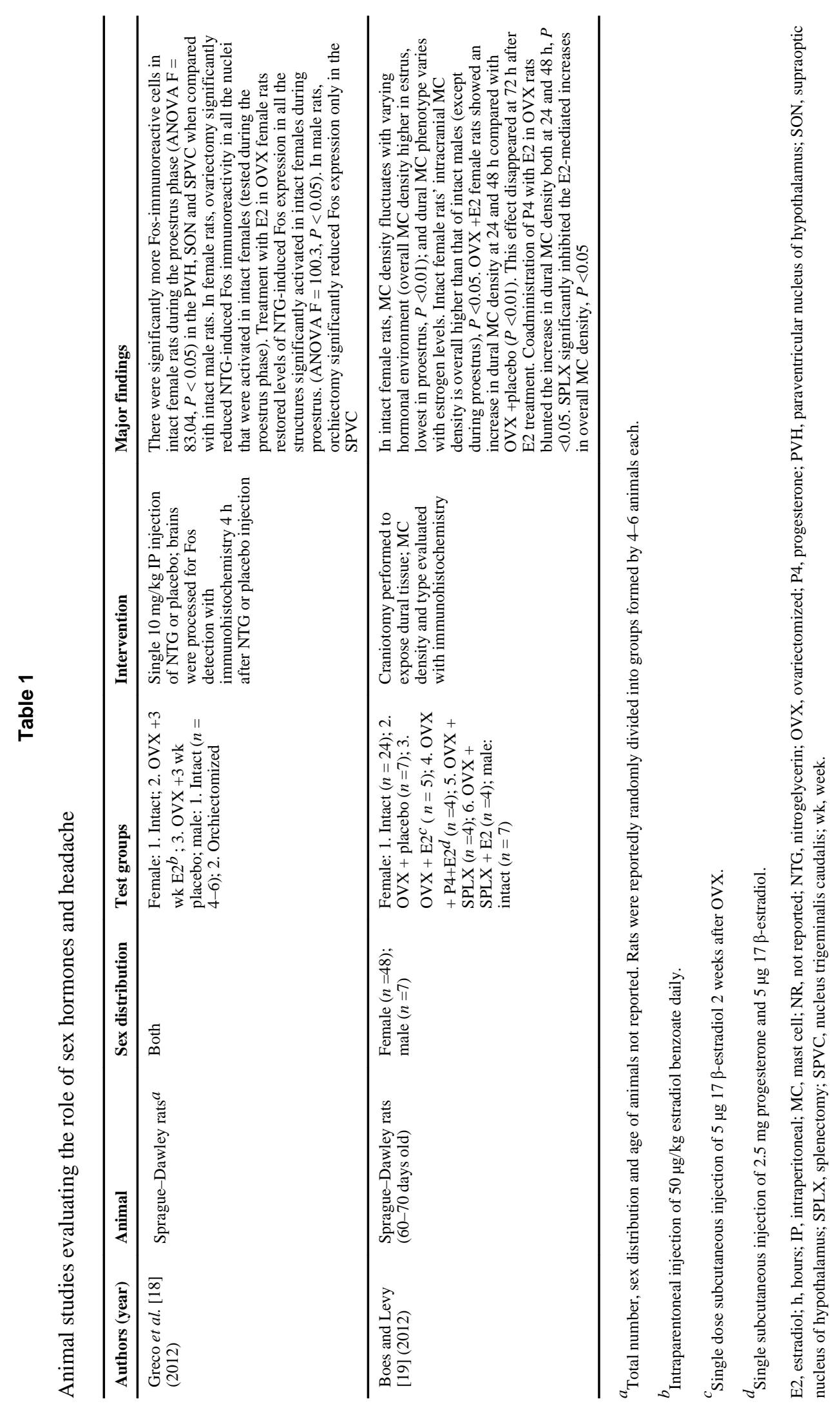




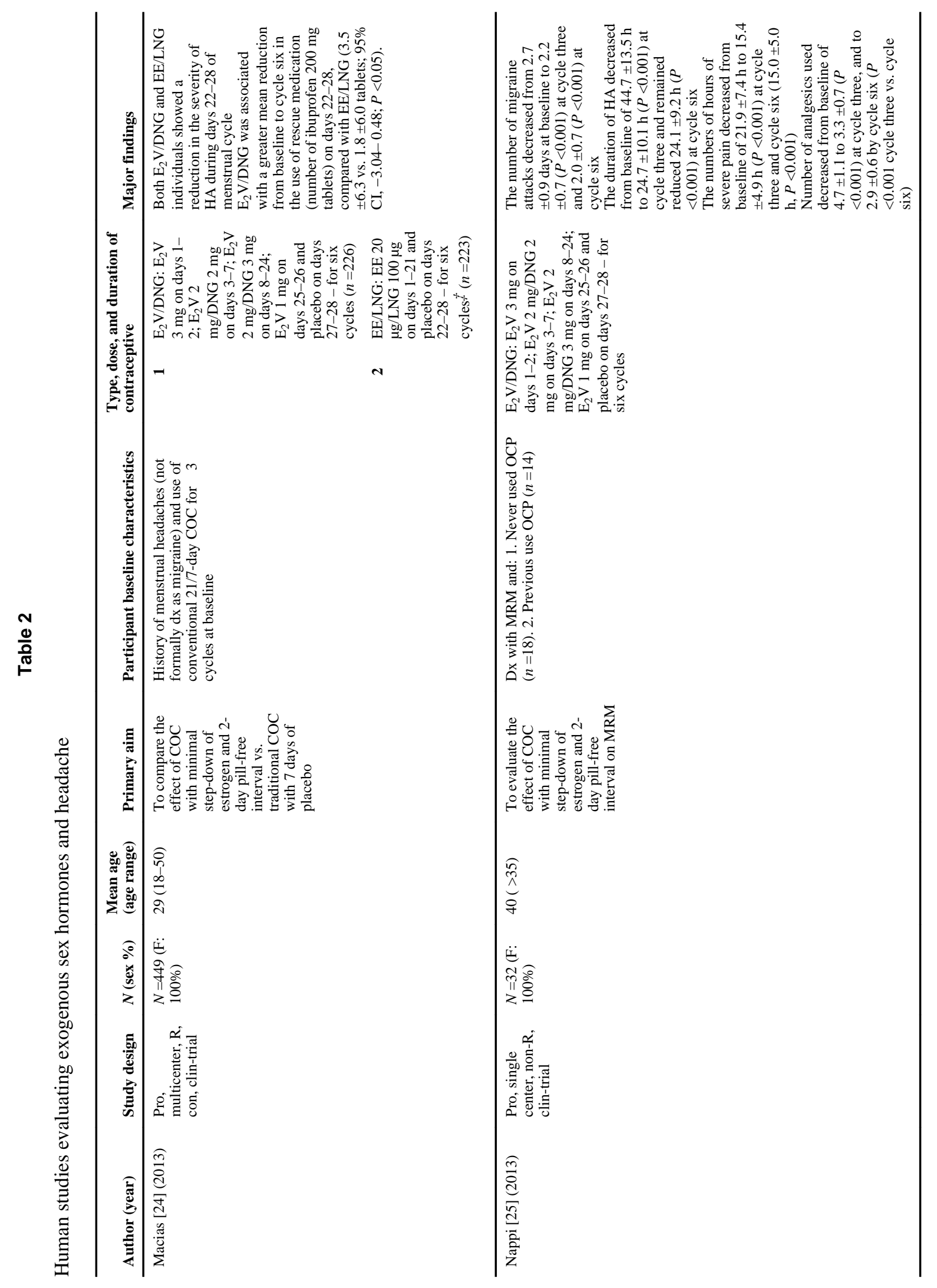




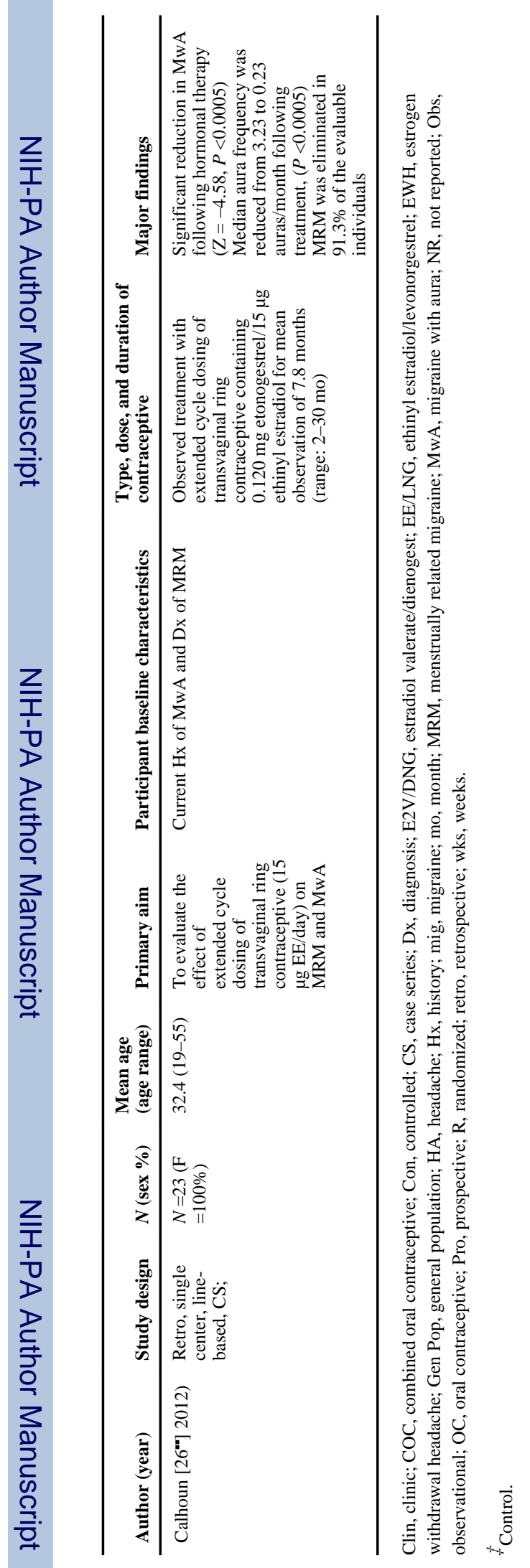

